\title{
Design, Synthesis and Biological Activities of Chalcones with Piperonal Moiety
}

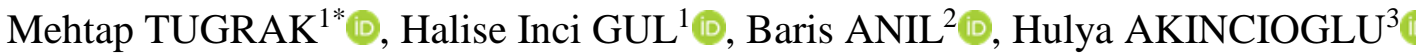 \\ ${ }^{1}$ Department of Pharmaceutical Chemistry, Faculty of Pharmacy, Ataturk University, Erzurum, Turkey; \\ ${ }^{2}$ Faculty of Science, Department of Chemistry, Ataturk University, Erzurum, Turkey; \\ ${ }^{3}$ Department of Chemistry, Faculty of Sciences and Arts, Agri Ibrahim Cecen University, Agri, Turkey
}

Geliş / Received: 04/03/2020, Kabul / Accepted: 30/05/2020

\begin{abstract}
Heterocyclic compounds are of specific significance between pharmacologically active compounds. In this study, some piperonal-based chalcones (PC1-PC10) were synthesized with Claisen-Schmidt Condensation with the reaction between 3,4-methylenedioxybenzaldehyde and several acetophenones. Inhibition potency of the chalcones were evaluated toward human carbonic anhydrase I and II enzymes (hCA I and hCA II), and acetylcholinesterase $(\mathrm{AChE})$. The chalcone derivatives were found to have $\mathrm{IC}_{50}$ values in the range of 5.11109.70 $\mu \mathrm{M}$ for hCA I, 17.05-162.59 $\mu \mathrm{M}$ for hCA II, and 18.52-98.69 $\mu \mathrm{M}$ for AChE. All compounds showed lower inhibition potential than reference compounds. While the PC3 (methoxy derivative) compound was the most effective compound against both hCA I and hCA II, PC5 (fluorine derivative) showed the strongest inhibition effect against AChE in the series. Results confirmed that the chalcone derivatives PC3 and PC5 can be considered as favorable candidates against hCA I, hCA II and AChE isoenzymes to design more potent enzyme inhibitors.
\end{abstract}

Keywords: Alzheimer's disease, chalcone, carbonic anhydrase, piperonal, acetylcholinesterase

\section{Piperonal Artığı Taşıyan Şalkonların Dizaynı, Sentezi ve Biyolojik Aktiviteleri}

\section{$\ddot{O} \mathbf{z}$}

Heterosiklik bileşikler, farmakolojik olarak aktif bileşikler arasında özel bir öneme sahiptir. Bu çalışmada, bazı piperonal bazlı şalkonlar (PC1-PC10), 3,4-metilendioksibenzaldehid ve birkaç asetofenon arasında Claisen-Schmidt Kondenzasyonu ile sentezlendi. Şalkonların inhibisyon potansiyelleri insan karbonik anhidraz I, II enzimlerine (hCA I ve hCA II) ve asetilkolinesteraz (AChE) enzimine karşı araştırıldı. Şalkon türevlerinin, hCA I için 5.11-109.70 $\mu \mathrm{M}$, hCA II için 17.05-162.59 $\mu \mathrm{M}$ ve AChE için 18.52-98.69 $\mu \mathrm{M}$ aralığında $\mathrm{IC}_{50}$ değerlerine sahip olduğu görüldü. Tüm bileşikler referans bileşiklerden daha düşük inhibisyon potansiyeli gösterdi. PC3 (metoksi türevi) bileşiği hem hCA I hemde hCA II'ye karşı en etkili bileşik olurken, PC5 (flor türevi) AChE'ye karşı seri içinde en güçlü inhibitör etki göstermiştir. Sonuçlar, şalkon türevleri PC3 ve PC5'in hCA I, hCA II ve AChE izoenzimlerine karşı daha güçlü enzim inhibitörleri tasarlamada uygun adaylar olarak kabul edilebileceğini doğrulamıştır.

Anahtar Kelimeler: Alzheimer Hastalığı, şalkon, karbonik anhidraz, piperonal, asetilkolinesteraz

\section{Introduction}

Alzheimer's disease (AD) is very prevalent kind of dementia in the aged, effecting approximately 50 million people worldwide according to the 2018 World Alzheimer's report. $\mathrm{AD}$ is

*Corresponding Author: mehtaptugrak@ hotmail.com qualified by the extracellular collecting of amyloid- $\beta(\mathrm{A} \beta)$ plaques and intracellular neurofibrillary tangles (NFTs) of hyper phosphorylated tau protein, related to neuronal cell death. AD is a multicomponent disease including different etiopathogenic mechanisms, for instance 
Amyloid Precursor Protein (APP) pathogenic division, mitochondrial dysfunction, oxidative stress, neurotransmitter dishomeostasis, protein misfolding, and neuronal abortive cell cycle re-entry (Nunez-Borque, et al., 2020). There is no clinically accepted treatment for the complete treatment and cessation of progression of AD. Presently, there are only five FDAcertified drugs such as Donepezil, Rivastigmine, Tacrine and Galantamine for treatment of this disease. These drugs involve cholinesterase inhibitors, $\mathrm{N}$ methyl-d-aspartate (NMDA) receptor antagonist (Hasan, et al., 2019). At the same time, these drugs have critical side effects on patients, but only provide temporary symptomatic relief.

Acetylcholinesterase enzyme (AChE, E.C.3.1.1.7) is liable for the degeneracy of acetylcholine $(\mathrm{ACh})$ and its inhibition result increase in ACh levels. In this respect, AChE inhibitors are acceptable one of the most valuable lines for AD's therapy. Commercially available drugs such as Exelon, Aricept and Razadyne serve as an antagonist of the enzyme acetylcholinesterase. These drugs cannot fully treat the $\mathrm{AD}$ while they raise the level of ACh in neurons so the cognitive function is enhanced (Shukla and Singh, 2020). Takrin and Donepezil of the same synthetic origin are used for the treatment of cognitive loss in patients with AD. Nevertheless, Tacrine's hepatotoxic effect require to searching and growing new members with significant safety. Therefore, many tacrine-like compounds were synthesized. Owing to the critical side effects of Takrine and Donepezil, the need for more selective anticholinesterase inhibitors is still compulsory (Anoja, et al., 2018).
Carbonic anhydrase (CA, E.C.4.2.1.1) enzymes regulate the $\mathrm{pH}$ of the living system and catalyze the two-sided hydration of carbon dioxide $\left(\mathrm{CO}_{2}\right)$ (Tugrak et al., 2018; Supuran, 2008). Carbonic anhydrase enzymes are frequently metallo enzymes containing zinc (II) in their active regions. The carbonic anhydrase enzyme has 16 isoenzymes, which are dispersed to almost all of body. Inhibition of these enzymes is investigated to examine the causes of many diseases, such as cancer and glaucoma (Tugrak et al., 2018; Supuran, 2008). Chalcones or 1,3diphenyl-2-propene-1-one can be attain from both the herbs (Bai et al., 2019; Gul et al., 2019; Tugrak et al., 2019; Tugrak et al., 2018; Yamali et al., 2017; Yamali et al., 2016). Chalcones are formed by condensation a ketone with aromatic aldehyde in acidic or basic conditions. The chalcones structures are composed by two aromatic rings bounded by an olefin portion and an $\alpha, \beta$-unsaturated carbonyl system. These compounds have many biological activities, for instance antioxidant (Diaz-Rubio et al., 2019), anti-inflammatory (Reddy et al., 2017), carbonic anhydrase inhibitory (Yamali et al., 2016), anticancer (Yamali et al., 2017; Yamali et al., 2016) and treatment of Alzheimer's disease (Diaz-Rubio et al., 2019; Tian et al., 2020). Because of the synthesis of $\mathrm{AChE}$ inhibitor compounds is important in the treatment of $\mathrm{AD}$, studies in this field have started to get speed recently. Chalcone derivatives are promising compounds to be developed as $\mathrm{AChE}$ and $\mathrm{CA}$ inhibitors. Piperonal is a heterocyclic ring system including a benzene ring fuzed to a pyran ring. The chalcone derivatives having piperonal moiety structure have various 
biological activities (Asrar and Hussain, 2018). In the light of literatures, we designed and synthesized (E)-3(benzo[d][1,3]dioxol-5-yl)-1-

substitutedphenylprop-2-en-1-ones and their structures were explained by way of ${ }^{1} \mathrm{H}$ NMR spectra. Besides, we have utilized inhibition possible of the chalcone compounds PC1-PC10 on AChE and hCA I and hCA II enzymes discover the most favorable inhibitors on the enzyme at issue. The results to be obtained may give directions to farther studies.

\section{Materials and Methods}

\subsection{Chemistry}

The chemical structures of the target compounds were affirmed by the Nuclear Magnetic Resonance (NMR) spectra ${ }^{1} \mathrm{H}$ NMR (400MHz), (Varian Mercury Plus spectrometer, Varian Inc., Palo Alto, California, U.S.). Chemical shifts $(\delta)$ are declared in ppm and coupling constants $(J)$ are denoted as hertz $(\mathrm{Hz})$. Melting points were specified using an Electrothermal 9100/IA9100 instrument (Bibby Scientific Limited, Staffordshire, UK) and are uncorrected. Reactions were monitored by Thin Layer Chromatography (TLC) using silica gel 60 HF254 (Merck KGaA). Chloroform: methanol (4.5:0.5) solvent mixture was used as TLC solvent systems. DMSO ${ }_{\mathrm{d} 6}$ (Merck) and $\mathrm{CDCl}_{3}$ were used as NMR solvents.

\section{General synthesis of (benzo[d][1,3]dioxol-5-yl)-1-}

\section{(substitutedphenyl)prop-2-en-1-one, compounds PC1-10, Figure 1}

To the mixture of 1,3-benzodioxole-5carboxaldehyde $(1 \mathrm{mmol})$ and a suitable acetophenone $(1 \mathrm{mmol})$ in $\mathrm{EtOH}(20$ $\mathrm{mL}$ ), $\mathrm{NaOH}$ (in aqua $10 \%, 4 \mathrm{~mL}$ ) was added. The contents of the flask were stirred at room temperature for 24 hours. The formed substance is poured into cold water $(100 \mathrm{~mL})$ and then $\mathrm{HCI}$ (in aqua $10 \%$, w/v) was added until mixture neutralized. The solid formed was filtered and washed with water. $\mathrm{H}_{2} \mathrm{O}$ /Ethanol mixture was used for crystallization (Tugrak et al., 2019; Tugrak et al., 2018; Gul et al., 2019; Yamali et al., 2017) (Figure 1).
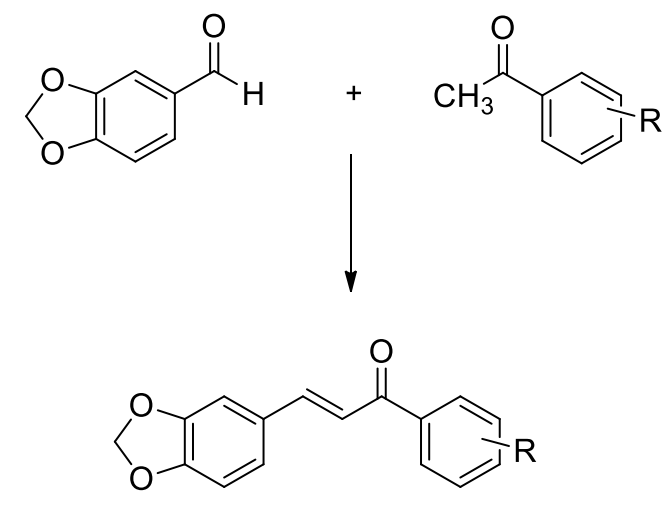

$$
\begin{aligned}
& \text { PC1; R=H, PC2; R=4- } \mathrm{CH}_{3} \text {, } \\
& \text { PC3; } \mathrm{R}=4-\mathrm{CH}_{3} \mathrm{O}, \mathrm{PC} 4 ; \mathrm{R}=4-\mathrm{Cl} \text {, } \\
& \text { PC5; R=4-F, PC6; R=4-Br, } \\
& \text { PC7; R=4-OH, 3- } \mathrm{CH}_{3} \mathrm{O}, \mathbf{P C 8} ; \mathrm{R}=3-\mathrm{OH} \text {, } \\
& \text { PC9; } \mathrm{R}=4-\mathrm{OH}, \mathbf{P C 1 0} ; \mathrm{R}=4-\mathrm{NO}_{2}
\end{aligned}
$$

Reagents: (i) $10 \% \mathrm{NaOH}$, EtOH, r.t.

Figure 1. Synthesis of the compounds PC1-PC10

\section{(E)-3-(Benzo[d][1,3]dioxol-5-yl)-1- phenylprop-2-en-1-one (PC1)}

Yield: 90\%. m.p.: $130-131^{\circ} \mathrm{C}$, Lit m.p.: 118$122^{\circ} \mathrm{C}$ (Jithan et al., 2009). ${ }^{1} \mathrm{H}-\mathrm{NMR}\left(\mathrm{CDCl}_{3}\right)$ $\delta 7.84$ (d, 1H, J=3.6 Hz, Ar-H), 7.77 (d, 1H, $J=15.4 \mathrm{~Hz},=\mathrm{CH}), 7.67(\mathrm{~d}, 1 \mathrm{H}, J=4.9 \mathrm{~Hz}, \mathrm{Ar}-$ $\mathrm{H})$, 7.28-7.24 (m, 3H, Ar-H), 7.19-7.12 (m, (E)-3- $\quad 3 \mathrm{H}, \mathrm{Ar}-\mathrm{H}), 6.85(\mathrm{~d}, 1 \mathrm{H}, J=8.0 \mathrm{~Hz}, \mathrm{Ar}-\mathrm{H})$, 6.03 (s, $2 \mathrm{H},-\mathrm{OCH}_{2} \mathrm{O}-$ ). 
(E)-3-(Benzo[d][1,3]dioxol-5-yl)-1-(ptolyl)prop-2-en-1-one (PC2)

Yield: $86 \%$. m.p.: $129-130^{\circ} \mathrm{C}$, Lit m.p.: $112^{\circ} \mathrm{C}$ (Pal, 2013). ${ }^{1} \mathrm{H}-\mathrm{NMR}\left(\mathrm{CDCl}_{3}\right) \delta 7.92$ (d, 2H, $J=8.2 \mathrm{~Hz}, \mathrm{Ar}-\mathrm{H}), 7.73$ (d, 1H, $J=15.5$ $\mathrm{Hz},=\mathrm{CH}), 7.37$ (d, 1H, J=15.6 Hz, =CH), 7.30-7.26 (m, 2H, Ar-H), 7.17 (s, 1H, Ar-H), 7.12 (dd, 1H, J=8.0 Hz, $1.6 \mathrm{~Hz}$, Ar-H), 6.84 (d, 1H, J=8.0 Hz, Ar-H), $6.02(\mathrm{~s}, 2 \mathrm{H},-$ $\left.\mathrm{OCH}_{2} \mathrm{O}-\right), 2.43$ (s, 3H, $\left.-\mathrm{CH}_{3}\right)$.

(E)-3-(Benzo[d][1,3]dioxol-5-yl)-1-(4methoxyphenyl)prop-2-en-1-one (PC3)

Yield: $85 \%$. m.p.: $134-135^{\circ} \mathrm{C}$, Lit m.p.: 135 $138^{\circ} \mathrm{C}$ (Jithan et al., 2009). ${ }^{1} \mathrm{H}-\mathrm{NMR}\left(\mathrm{CDCl}_{3}\right)$ $\delta 8.02(\mathrm{~d}, 2 \mathrm{H}, J=8.8 \mathrm{~Hz}, \operatorname{Ar}-\mathrm{H}), 7.72(\mathrm{~d}, 1 \mathrm{H}$, $J=15.5 \mathrm{~Hz},=\mathrm{CH}), 7.38(\mathrm{~d}, 1 \mathrm{H}, J=15.5 \mathrm{~Hz}$, $=\mathrm{CH}), 7.17(\mathrm{~s}, 1 \mathrm{H}, \mathrm{Ar}-\mathrm{H}), 7.12(\mathrm{dd}, 1 \mathrm{H}$, $J=8.0 \mathrm{~Hz}, 1.6 \mathrm{~Hz}, \mathrm{Ar}-\mathrm{H}), 6.98(\mathrm{~d}, 2 \mathrm{H}, J=8.8$ $\mathrm{Hz}, \mathrm{Ar}-\mathrm{H}), 6.84$ (d, 1H, J=8.0 Hz, Ar-H), 6.02 (s, $\left.2 \mathrm{H},-\mathrm{OCH}_{2} \mathrm{O}-\right), 3.89$ (s, $\left.3 \mathrm{H},-\mathrm{CH}_{3} \mathrm{O}\right)$.

(E)-3-(Benzo[d][1,3]dioxol-5-yl)-1-(4chlorophenyl)prop-2-en-1-one (PC4)

Yield: $80 \%$. m.p.: $128-129^{\circ} \mathrm{C}$, Lit m.p.: $123-$ $128^{\circ} \mathrm{C}$ (Jithan et al., 2009). ${ }^{1} \mathrm{H}-\mathrm{NMR}\left(\mathrm{CDCl}_{3}\right)$ $\delta 7.95(\mathrm{~d}, 2 \mathrm{H}, J=8.6 \mathrm{~Hz}, \mathrm{Ar}-\mathrm{H}), 7.73(\mathrm{~d}, 1 \mathrm{H}$, $J=15.6 \mathrm{~Hz},=\mathrm{CH}), 7.47(\mathrm{~d}, 2 \mathrm{H}, J=8.5 \mathrm{~Hz}, \mathrm{Ar}-$ $\mathrm{H}), 7.32(\mathrm{~d}, 1 \mathrm{H}, J=15.5 \mathrm{~Hz},=\mathrm{CH}), 7.17(\mathrm{~s}$, 1H, Ar-H), 7.12 (dd, 1H, J=8.0 Hz, $1.6 \mathrm{~Hz}$, Ar-H), 6.85 (d, 1H, J=8.0 Hz, Ar-H), 6.03 (s, $\left.2 \mathrm{H},-\mathrm{OCH}_{2} \mathrm{O}-\right)$.

\section{(E)-3-(Benzo[d][1,3]dioxol-5-yl)-1-(4-} fluorophenyl)prop-2-en-1-one (PC5)

Yield: $85 \%$. m.p.: ${ }^{136}-137^{\circ} \mathrm{C} .{ }^{1} \mathrm{H}-\mathrm{NMR}$ $\left(\mathrm{CDCl}_{3}\right) \delta$ 8.06-8.02 (m, 2H, Ar-H), $7.74(\mathrm{~d}$, $1 \mathrm{H}, J=15.5 \mathrm{~Hz},=\mathrm{CH}), 7.34(\mathrm{~d}, 1 \mathrm{H}, J=15.5$ $\mathrm{Hz},=\mathrm{CH}), 7.19-7.11(\mathrm{~m}, 4 \mathrm{H}, \mathrm{Ar}-\mathrm{H}), 6.85$ (d, $1 \mathrm{H}, J=8.0 \mathrm{~Hz}, \mathrm{Ar}-\mathrm{H}), 6.03$ (s, 2H, $-\mathrm{OCH}_{2} \mathrm{O}-$ ).
(E)-3-(Benzo[d][1,3]dioxol-5-yl)-1-(4bromophenyl)prop-2-en-1-one (PC6)

Yield: 83\%. m.p.: $137-138^{\circ} \mathrm{C}$, Lit m.p.: 111$113^{\circ} \mathrm{C}$ (Pathak et al., 2003). ${ }^{1} \mathrm{H}-\mathrm{NMR}$ $\left(\mathrm{CDCl}_{3}\right) \delta 7.87(\mathrm{~d}, 2 \mathrm{H}, J=8.5 \mathrm{~Hz}, \mathrm{Ar}-\mathrm{H}), 7.75$ $(\mathrm{d}, 1 \mathrm{H}, J=15.6 \mathrm{~Hz},=\mathrm{CH}), 7.62(\mathrm{~d}, 2 \mathrm{H}, J=8.5$ $\mathrm{Hz}, \operatorname{Ar}-\mathrm{H}), 7.35(\mathrm{~d}, 1 \mathrm{H}, J=15.5 \mathrm{~Hz},=\mathrm{CH})$, $7.16(\mathrm{~s}, 1 \mathrm{H}, \mathrm{Ar}-\mathrm{H}), 7.12(\mathrm{dd}, 1 \mathrm{H}, J=8.0 \mathrm{~Hz}$, $1.6 \mathrm{~Hz}, \mathrm{Ar}-\mathrm{H}), 6.85$ (d, 1H, J=8.0 Hz, Ar-H), 6.03 (s, $2 \mathrm{H},-\mathrm{OCH}_{2} \mathrm{O}-$ ).

(E)-3-(Benzo[d][1,3]dioxol-5-yl)-1-(4hydroxy-3-methoxyphenyl)prop-2-en-1-one (PC7)

Yield: $70 \%$. m.p.: $166-168^{\circ} \mathrm{C}$, Lit m.p.: 126$128^{\circ} \mathrm{C}$ (Reddy et al., 2017). ${ }^{1} \mathrm{H}-\mathrm{NMR}$ $\left(\mathrm{CDCl}_{3}\right) \delta 9.80(\mathrm{~s}, 1 \mathrm{H}, \mathrm{OH}), 7.71(\mathrm{~d}, 1 \mathrm{H}$, $J=11.4 \mathrm{~Hz},=\mathrm{CH}), 7.59$ (s, 1H, Ar-H), 7.53 (s, 1H, Ar-H), $7.46(\mathrm{~d}, 1 \mathrm{H}, J=11.4 \mathrm{~Hz},=\mathrm{CH})$, $7.24(\mathrm{~d}, 1 \mathrm{H}, J=8.1 \mathrm{~Hz}, \operatorname{Ar}-\mathrm{H}), 6.95(\mathrm{~d}, 1 \mathrm{H}$, $J=8.0 \mathrm{~Hz}, \mathrm{Ar}-\mathrm{H}), 6.50-6.30$ (m, 2H, Ar-H), $6.08\left(\mathrm{~s}, 2 \mathrm{H},-\mathrm{OCH}_{2} \mathrm{O}-\right), 3.78\left(\mathrm{~s}, 3 \mathrm{H},-\mathrm{CH}_{3} \mathrm{O}\right)$.

\section{(E)-3-(Benzo[d][1,3]dioxol-5-yl)-1-(3-} hydroxyphenyl)prop-2-en-1-one (PC8)

Yield: $82 \%$. m.p.: $183-185^{\circ} \mathrm{C} .{ }^{1} \mathrm{H}-\mathrm{NMR}$ (DMSO-d6) $\delta 10.40(\mathrm{~s}, 1 \mathrm{H}, \mathrm{OH}), 7.73(\mathrm{~d}, 1 \mathrm{H}$, $J=15.6 \mathrm{~Hz},=\mathrm{CH}), 7.66-7.60(\mathrm{~m}, 3 \mathrm{H}, \mathrm{Ar}-\mathrm{H}$, $=\mathrm{CH}), 7.46(\mathrm{~s}, 1 \mathrm{H}, \mathrm{Ar}-\mathrm{H}), 7.37-7.30(\mathrm{~m}, 2 \mathrm{H}$, Ar-H), 7.05 (d, 1H, J=8.0 Hz, Ar-H), 6.98 (d, $1 \mathrm{H}, J=8.0 \mathrm{~Hz}, \mathrm{Ar}-\mathrm{H}), 6.10$ (s, 2H, $-\mathrm{OCH}_{2} \mathrm{O}-$ ).

\section{(E)-3-(Benzo[d][1,3]dioxol-5-yl)-1-(4-} hydroxyphenyl)prop-2-en-1-one (PC9)

Yield: $80 \%$. m.p.: $183-185^{\circ} \mathrm{C} .{ }^{1} \mathrm{H}-\mathrm{NMR}$ (DMSO-d6) $\delta 10.40$ (s, 1H, OH), 8.07 (d, 2H, $J=8.6 \mathrm{~Hz}, \mathrm{Ar}-\mathrm{H}), 7.80(\mathrm{~d}, 1 \mathrm{H}, J=15.5 \mathrm{~Hz}$, $=\mathrm{CH}), 7.63-7.59$ (m, 2H, Ar-H, =CH), 7.27 (d, 1H, J=8.0 Hz, Ar-H), 6.98 (d, 1H, J=8.0 $\mathrm{Hz}, \operatorname{Ar}-\mathrm{H}), 6.88$ (d, 2H, J=8.7 Hz, Ar-H), $6.10\left(\mathrm{~s}, 2 \mathrm{H},-\mathrm{OCH}_{2} \mathrm{O}\right)$. 
(E)-3-(Benzo[d][1,3]dioxol-5-yl)-1-(4nitrophenyl)prop-2-en-1-one (PC10)

Yield: $60 \%$. m.p.: $199-200^{\circ} \mathrm{C}$, Lit m.p.: 203$205^{\circ} \mathrm{C}$ (Chiaradia et al., 2008). ${ }^{1} \mathrm{H}-\mathrm{NMR}$ $\left(\mathrm{DMSO}_{-\mathrm{d} 6}\right) \delta 7.85(\mathrm{~d}, 1 \mathrm{H}, J=15.5 \mathrm{~Hz},=\mathrm{CH})$, 7.76 (s, 1H, Ar-H), 7.72-7.68 (m, 3H, Ar-H, $=\mathrm{CH}), 7.37(\mathrm{~d}, 2 \mathrm{H}, J=8.0 \mathrm{~Hz}, \mathrm{Ar}-\mathrm{H}), 7.01(\mathrm{~d}$, $2 \mathrm{H}, J=8.0 \mathrm{~Hz}, \mathrm{Ar}-\mathrm{H}), 6.13$ (s, $2 \mathrm{H},-\mathrm{OCH}_{2} \mathrm{O}-$ ).

\subsection{Biological Activity}

\subsubsection{AChE inhibition study}

As mentioned in the literature, AChE activities of the compounds were quantified by changing the spectrophotometric procedure (Burmaoglu et al., 2018; Ozgun et al., 2016; Timur et al., 2019; Yamali et al., 2018). While using acetylthiocoline iodide (AChI) as the substrate, 5,5-dithiobis (2nitro-benzoic acid) (DTNB) was utilized to detection of the activity.

Shortly, Tris/HCl buffer (100 mL, 1M, $\mathrm{pH}=8)$ and sample solution $(10 \mathrm{~mL})$ dissolved in ultra pure water at different concentrations and AChE solution $(50 \mathrm{~mL})$ were added and the mixture was incubated for $10 \mathrm{~min}$ at $25^{\circ} \mathrm{C}$. DTNB $(50 \mathrm{~mL}, 0.5 \mathrm{mM})$ was added on it. AChI (50 mL, $10 \mathrm{mM})$ was added to start the reaction. The hydrolysis of substrates was evaluated at $412 \mathrm{~nm}$. The $\mathrm{IC}_{50}$ values were calculated using an activity (\%)[Compound] graph. The enzyme assay was repeated three times. Tacrine was used a reference compound.

\subsection{2. hCA inhibition studies}

Sepharose-4B-L-Tyrosine- sulfanilamide affinity chromatography were used for the purification of CA isoforms using fresh human blood erythrocytes. Erythrocytes samples were centrifuged at $16,000 x$ for 30 min and the buffy coat and plasma were discarded. $\mathrm{pH}$ of buffer was adjusted to 8.7 by solid Tris (Akbaba et al., 2014). After centrifugation, supernatant was transferred to the Sepharose- 4B-L-Tyrosine- sulfanilamide affinity column. The enzymes were spectrophotometrically measured at $280 \mathrm{~nm}$. Sodium dodecyl sulfate-polyacrylamide gel electrophoresis (SDS-PAGE) was carried out for registration of both isoforms purity. Human CA isoforms activity designation was observed spectrophotometrically (Shimadzu, UV-VIS Spectrophotometer, UVmini- 1240, Kyoto, Japan) (Verpoorte et al., 1967). In this test, variations in absorbance were obtained during $3 \mathrm{~min}$ at $30^{\circ} \mathrm{C}$. p-Nitrophenylacetate (NPA) molecule was used as substrate. Protein quantity was identified by Bradford process (Bradford 1976). The bovine serum albumin was used as a control at $595 \mathrm{~nm}$. For obtaining the inhibition results of each isoform, the compounds PC1-PC10 and an activity (\%) [PC1-PC10] graph were drawn. The Lineweaver-Burk curves were drawn and $\mathrm{IC}_{50}$ calculations were realised (Lineweaver and Burk, 1934). Acetazolamide was used as a control drug.

\section{Result and Discussion}

\subsection{Chemistry}

Chalcones PC1-PC10 were prepared by Claisen-Schmidt Condensation of suitable acetophenones [acetophenone (PC1), 4methylacetophenone(PC2),4methoxyacetoph enone (PC3), 4-chloroacetophenone (PC4), 4-fluoroacetophenone(PC5),4 bromoacetophenone (PC6), 4-hydroxy-3methoxyacetophenone (PC7), 3hydroxyacetophenone (PC8), 4hydroxyacetophenone (PC9), 4nitroacetophenone (PC10)], reacted with 3,4methylenedioxybenzaldehyde in equimolar 
quantities alkaline condition $(\mathrm{NaOH})$ at room temperature (Figure 1). The reaction proceed was checked by TLC monitoring. Later, the reaction content was taken into ice water and acidified with $\mathrm{HCl}$ (aqua 10\%) until $\mathrm{pH}=7$. Ethanol-water mixture was utilized for crystallization. Since all compounds were previously registered in the literature their structures were confirmed here by their melting points and ${ }^{1} \mathrm{H}$ NMR spectra. Characterization of the chalcones PC1-PC10 by ${ }^{1} \mathrm{H}$ NMR was presented in the experimental part in detail.

Chalcones are open-chain flavonoids. Chemically they consist of $\alpha, \beta$-unsaturated carbonyl system and two aromatic rings. They are available in both cis $(Z)$ and trans $(E)$ forms. In this work, the compounds were seen as $\mathrm{E}$ isomer on the basis of coupling constants. Olefinic carbon-carbon bond of each chalcone was established by their large trans coupling constants $(\mathrm{J})$ in the range of ${ }^{15}-16 \mathrm{~Hz}$ in the ${ }^{1} \mathrm{H}$ NMR spectra. The characteristic methylenedioxy protons ($\left.\mathrm{OCH}_{2} \mathrm{O}-\right)$ in the piperonal chalcone derivatives were observed as singlet between 6.02 and $6.13 \mathrm{ppm}$ in ${ }^{1} \mathrm{H}$ NMR.

\subsection{Acetylcholinesterase inhibitory activity}

To see whether the chalcone (PC1-PC10) compounds have AChE inhibitory features, the compounds (PC1-PC10) were investigated against AChE enzyme (Table 1). A cholinergic enzyme inhibition property was recorded based on the method of Ellman et al. (Ellman, Courtney, Andres, \& FeatherStone, 1961). Chalcones derivatives had $\mathrm{IC}_{50}$ values in range of 18.52-98.69 $\mu \mathrm{M}$ for AChE. Besides, Tacrine had $\mathrm{IC}_{50}$ value of $0.38 \mu \mathrm{M}$. All evaluated chalcones derivatives
(PC1-PC10) showed lower inhibition than reference compound against $\mathrm{AChE}$.

When the halogen substituted derivatives [PC4 (chlorine), PC5 (fluorine), PC6 (bromine)] were considered, all three halogenated compounds had more potent inhibition profile than $\mathrm{PC} 1$, which is nonsubstituted derivative. Among them PC5 $(18.52 \mu \mathrm{M})$ which is fluorine substituted compound had the highest inhibition potency on AChE among the three compounds with the lowest $\mathrm{IC}_{50}$ value. So, it seems that converting the nonsubstituted derivative PC1 to halogenated derivative was beneficial and efficient molecular modification based on the $\mathrm{IC}_{50}$ value. When halogen groups were added to position 4 , it had a positive effect on increasing the inhibition potency on AChE. When fluorine, bromine and chlorine atoms were compared, the fluorine, which has smaller atomic diameter than the others. The most potent inhibition profile on $\mathrm{AChE}$ enzyme suggestion the importance of size of atom inserted.

When AChE inhibitory effects of the monohydroxy substituted derivatives (PC8, PC9) were considered, the compound PC8 with 3-hydroxyphenyl $(54.57 \mu \mathrm{M})$ had more potent inhibition compound than the 4hydroxyphenyl derivative $(68.21 \mu \mathrm{M})$.

When 4-nitro substituted derivatives were considered, it was seen that nitro group, which is an electron-attracting group, had no inhibitory effect on AChE enzyme.

The leader compound of the series is PC5, which has fluorine substitution on 4 th position of the phenyl ring.

It was mentioned in the introduction part that chalcones have a wide variety of bioactivities. It was reported that many 
enzymes which include AChE (Burmaoglu et al., 2020) were inhibited by the chalcones. In previous study (Burmaoglu et al., 2020), new organohalogen chalcone derivatives (5-12) were analyzed against acetylcholinesterase (AChE) enzymes. These compounds (5-12) displayed $\mathrm{IC}_{50}$ value in range of 2.97-5.72 $\mathrm{nM}$ on AChE. According to inhibition results, the new chalcone derivatives (5-12) had impressive inhibition potency.

The chalcone having piperonal moiety did not reported previously with AChE enzyme profile. We did basic comparison with the results reported in literature (Burmaoglu et al., 2020). Organohalogen chalcone derivatives (5-12) were found more powerful inhibitor than our compounds. Even we reported some halogenated compounds, their results were not attarctive than the paper above. It shows that the free methoxy substitution positively affected the bioactivity. So, using piperonal moiety instead of free methoxy substituents was not favorable modification to obtain significant results.

\subsection{Carbonic anhydrase I and II inhibition}

Carbonic anhydrase isoforms (hCA I and hCA II) are omnipresent. The inhibitors targeting these two isoenzymes can be beneficial candidates for the treatment of some diseases for instance glaucoma or epilepsy. In this research, we evaluated the effects of (E)-3-(benzo[d][1,3]dioxol-5-yl)-1substitutedphenylprop-2-en-1-ones (PC1PC10) against hCA I, hCA II. The CA inhibitor consequences of the compounds are presented in Table1.

Accordingly to our results, cytosolic isoform hCA I was inhibited by the investigated PC1PC10 chalcone derivatives with $\mathrm{IC}_{50}$ values ranging between 5.11 and $109.70 \mu \mathrm{M}$. Furthermore, PC3 (methoxy), PC4 (chlorine), and PC5 (fluorine) shown the most potent hCA I isoenzyme inhibition properties with $\mathrm{IC}_{50}$ values of 5.11, 12.97 and $13.48 \mu \mathrm{M}$, respectively. Reference drug acetazolamide (AZA) shown a $\mathrm{IC}_{50}$ value of $0.38 \mu \mathrm{M}$ (Table 1) against hCA I isoenzyme. It is understood that converting the nonsubstituted derivative PC1 to 4-methoxy substituted derivative (PC3), which is an electron-donating group, was beneficial and impressive molecular modification depends on the $\mathrm{IC}_{50}$ value $(5.11 \mu \mathrm{M})$ toward hCA I.

When the halogen substituted derivatives [PC4 (chlorine), PC5 (fluorine), PC6 (bromine)] were considered, 4-chlorophenyl derivative PC4 $(12.97 \mu \mathrm{M})$ had more potent inhibition than others halogenated compounds PC5 and PC6 toward hCA I with the lowest $\mathrm{IC}_{50}$ value.

As presented in Table 1, the inhibition profile of the considered chalcone derivatives (PC1PC10) against hCA II brought to light to be pretty similar to that indicated towards hCA

II. They shown $\mathrm{IC}_{50}$ values between 17.05$162.59 \mu \mathrm{M}$ against hCA II. PC3 (having a meyhoxy group), demonstrated a strongest inhibition effect toward hCA II with $\mathrm{IC}_{50}$ value $(17.05 \mu \mathrm{M})$. On the other hand, AZA had an $\mathrm{IC}_{50}$ value of $0.037 \mu \mathrm{M}$ toward hCA II.

When hydroxy substituted derivatives (PC8, PC9) were considered, it was shown that hydroxy group, which is an electron donating group, had no inhibitory effect on hCA I, and hCA II isoenzymes.

At the same time, carbonic anhydrase activities of chalcone derivatives were also investigated against human carbonic anhydrase I (hCA I), and carbonic anhydrase 
II (hCA II) enzymes by Burmaoğlu et al. These compounds (5-12) showed $\mathrm{IC}_{50}$ value in range of 2.07-35.0 nM on hCA I, 28.17$38.08 \mathrm{nM}$ on hCA II. According to inhibition results, the novel organohalogen chalcone derivatives (5-12) had more effective inhibition profiles than our results.

For future concept, we plan to synthesize sulfonamide derivatives as the most popular CA pharmacophore by using chalcones. We hope to get better CA enzyme inhibitory results with the sulfonamides derivatives.

\section{Conclusion}

As a result, this study includes the synthesis of piperonal bearing chalcones PC1-PC10 and evaluation of them in terms of inhibition potency toward hCA I, hCA II, and AChE. Although, all compounds showed lower inhibition potential than reference compounds, PC3 (methoxy) toward hCA I, and hCA II and PC5 (fluorine) toward AChE were candidate compounds of the series to develop new more potent compounds at issue.

\section{Conflicts of Interest}

There are no known conflicts of interest relevant this paper.

\section{Table Captions and Figure Legends}

Figure 1. Synthesis of the compounds PC1PC10

Table 1. Inhibition results of new piperonal chalcones (PC1-PC10) toward hCA I, hCA II and $\mathrm{AChE}$
Table 1. Inhibition results of new piperonal chalcones (PC1-PC10) toward hCA I, hCA II and $\mathrm{AChE}$

\begin{tabular}{|c|c|c|c|c|c|c|}
\hline & \multicolumn{2}{|c|}{ hCA-I } & \multicolumn{2}{|c|}{ hCA-II } & \multicolumn{2}{|c|}{$\mathrm{AChE}$} \\
\hline & $\mathrm{IC}_{50}(\mu \mathrm{M})$ & $\mathrm{r}^{2}$ & $\mathrm{IC}_{50}(\mu \mathrm{M})$ & $r^{2}$ & $\mathrm{IC}_{50}(\mu \mathrm{M})$ & $r^{2}$ \\
\hline $\mathrm{PC1}$ & 27.05 & 0.9156 & 54.39 & 0.9502 & 57.37 & 0.9699 \\
\hline $\mathrm{PC2}$ & 55.70 & 0.9342 & 39.02 & 0.9415 & 62.26 & 0.8742 \\
\hline $\mathrm{PC} 3$ & 5.11 & 0.9502 & 17.05 & 0.9451 & 56.07 & 0.8929 \\
\hline PC4 & 12.97 & 0.8952 & 95.94 & 0.9757 & 49.39 & 0.9425 \\
\hline PC5 & 13.48 & 0.9696 & 79.69 & 0.9402 & 18.52 & 0.9127 \\
\hline $\mathrm{PC} 6$ & 33.67 & 0.9702 & 49.57 & 0.9717 & 22.72 & 0.8831 \\
\hline $\mathrm{PC7}$ & 109.70 & 0.8794 & 162.59 & 0.8943 & 98.69 & 0.9884 \\
\hline PC8 & \multirow{2}{*}{\multicolumn{2}{|c|}{ nd }} & \multirow{2}{*}{\multicolumn{2}{|c|}{ nd }} & 54.57 & 0.9746 \\
\hline $\mathrm{PC} 9$ & & & & & 68.21 & 0.9377 \\
\hline $\mathrm{PC} 10$ & 91.35 & 0.9913 & 148.55 & 0.8296 & \multicolumn{2}{|c|}{ nd } \\
\hline Acetazolamide & 0.38 & 0.955 & 0.037 & 0.833 & $\cdot$ & $\cdot$ \\
\hline Tacrine & . & $\cdot$ & . & . & 0.38 & 0.989 \\
\hline
\end{tabular}

nd: Not detected

\section{References}

Akbaba, Y., Akincioglu, A., Gocer, H., Goksu, S., Gulcin, I., \& Supuran, C. T. 2014. "Carbonic anhydrase inhibitory properties of novel sulfonamide derivatives of aminoindanes and aminotetralins", Journal of Enzyme Inhibition and Medicinal Chemistry, 29(1), 35-42.

Attanayake, A.P., Jayatilaka, K. A. P. W. 2018. "Acetylcholinesterase Inhibitory Activity And Antioxidant Activity of Selected Medicinal Plant Extracts Used Against Cognitive Dysfunction And Memory Loss In Sri Lankan Traditional Medicine", Asian Journal of Pharmaceutical Research and Development, 6(5), 16-25.

Asrar A.A., Hussain, M. S. M. M. 2018. "Synthesis And Evaluation Of Piperonal 
Chalcone And Its Derivatives As AntiDiabetic Agents", European Journal of Biomedical and Pharmaceutical sciences, 5(2), 514-518.

Bai, P., Wang, K. R., Zhang, P. F., Shi, J., Cheng, X. F., Zhang, Q., Sang, Z. P. 2019. "Development of chalcone-O-alkylamine derivatives as multifunctional agents against Alzheimer's disease", European Journal of Medicinal Chemistry, 183, 111737

Bradford, M. M. 1976. "A rapid and sensitive method for the quantitation of microgram quantities of protein utilizing the principle of protein-dye binding", Analytic Biochemistry, $72,248-254$.

Burmaoglu, S., Yilmaz, A. O., Taslimi, P., Algul, O., Kilic, D., Gulcin, I. 2018. "Synthesis and biological evaluation of phloroglucinol derivatives possessing alphaglycosidase, acetylcholinesterase, butyrylcholinesterase, carbonic anhydrase inhibitory activity". Archive der Pharmazie (Weinheim), 351(2). doi: 10.1002/ardp.201700314

Burmaoglu, S., Kazancioglu, E., Kaya, R., Kazancioglu, M., Karaman, M., Algul, O., Gulcin, I. 2020.' 'Synthesis of novel organohalogen chalcone derivatives and screening of their molecular docking study and some enzymes inhibition effects". Journal of Molecular Structure 1208,127868.

Chiaradia, L. D., Mascarello, A., Purificacao, M., Vernal, J., Cordeiro, M. N. S., Zenteno, M. E., Terenzi, H. 2008. "Synthetic chalcones as efficient inhibitors of Mycobacterium tuberculosis protein tyrosine phosphatase PtpA", Bioorganic \& Medicinal Chemistry Letters, 18(23), 6227-6230.
Diaz-Rubio, L., Hernandez-Martinez, R., Estolano-Cobian, A., Chavez-Velasco, D., Salazar-Aranda, R., de Torres, N. W., Cordova-Guerrero, I. 2019. "Synthesis, Biological Evaluation and Docking Studies of Chalcone and Flavone Analogs as Antioxidants and Acetylcholinesterase Inhibitors", Applied Sciences-Basel, 9(3). doi: ARTN 410 10.3390/app9030410

Ellman, G. L., Courtney, K. D., Andres, V., Jr., Feather-Stone, R. M. 1961. "A new and rapid colorimetric determination of acetylcholinesterase activity". Biochemical Pharmacology, 7, 88-95.

Gul, H. I., Tugrak, M., Gul, M., Mazlumoglu, S., Sakagami, H., Gulcin, I., \& Supuran, C. T. 2019. "New phenolic Mannich bases with piperazines and their bioactivities", Bioorganic Chemistry, 90, 103057.

Lineweaver, H., Burk, D. 1934. "The determination of enzyme dissociation constants", Journal of American Chemical Society, 56, 658-666.

Hasan, A. H., Amran, S. I., Hussain, F. H. S., Jaff, B. A., Jamalis, J. 2019. "Molecular Docking and Recent Advances in the Design and Development of Cholinesterase Inhibitor Scaffolds:CoumarinHybrids",

Chemistryselect, 4(48), 14140-14156.

Jithan, A.V., Keerthana, E., Venkanna, P., Venumadhav, K., Reddy, K. S. 2009. "Synthesis of novel piperonal derivatives and evaluation of their anticonvulsant activity using a nanoparticular formulation", International journal of pharmaceutical sciences and nanotechnology, 2(1), 435-442. 
Nunez-Borque, E., Gonzalez-Naranjo, P., Bartolome, F., Alquezar, C., ReinaresSebastian, A., Perez, C., Martin-Requero, A. 2020. "Targeting Cannabinoid Receptor Activation and BACE-1 Activity Counteracts TgAPP Mice Memory Impairment and Alzheimer's Disease Lymphoblast Alterations", Molecular Neurobiology. doi: 10.1007/s 12035-019-01813-4

Ozgun, D. O., Yamali, C., Gul, H. I., Taslimi, P., Gulcin, I., Yanik, T., Supuran, C. T. 2016. "Inhibitory effects of isatin Mannich bases on carbonic anhydrases, acetylcholinesterase, and butyrylcholinesterase", Journal of Enzyme Inhibition and Medicinal Chemistry, 31(6), 1498-1501.

Pal, R. 2013. "Ammonium chloride catalyzed microwave-assisted Claisen-Schmidt reaction between ketones and aldehydes under solvent-free conditions", IOSR Journal of Applied Chemistry, 3(4), 74-80.

Pathak, V. N., Oza, C. K., Gupta, R., Tiwari, R., Chaudhary, S. 2003. "Synthesis and spectral studies of some new bis(1,3diketonato)palladium(II) complexes", Synthesis and Reactivity in Inorganic and Metal-Organic Chemistry, 33(4), 607-624.

Reddy, M. V. B., Hung, H. Y., Kuo, P. C., Huang, G. J., Chan, Y. Y., Huang, S. C., Wu, T. S. 2017. "Synthesis and biological evaluation of chalcone, dihydrochalcone, and 1,3-diarylpropane analogs as antiinflammatory agents", Bioorganic \& Medicinal Chemistry Letters, 27(7), 15471550.

Shukla, R., Singh, T. R. 2020. "Virtual screening, pharmacokinetics, molecular dynamics and binding free energy analysis for small natural molecules against cyclindependent kinase 5 for Alzheimer's disease", Journal of Biomolecular Structure \& Dynamics, 38(1), 248-262.

Supuran, C. T. 2008. "Carbonic anhydrases: novel therapeutic applications for inhibitorsand activators", Nature Reviews Drug Discovery, 7, 168-181.

Tian, C. Q., Qiang, X. M., Song, Q., Cao, Z. C., Ye, C. Y., He, Y. X., Zhang, L. 2020. "Flurbiprofen-chalcone hybrid Mannich base derivatives as balanced multifunctional agents against Alzheimer's disease: Design, synthesis and biological evaluation", Bioorganic Chemistry, 94. doi: ARTN 10347710.1016/j.bioorg.2019.103477

Timur, I., Kocyigit, U. M., Dastan, T., Sandal, S., Ceribasi, A. O., Taslimi, P., . . . Ciftci, M. 2019. "In vitro cytotoxic and in vivo antitumoral activities of some aminomethyl derivatives of 2,4-dihydro-3H1,2,4-triazole-3-thiones-Evaluation of their acetylcholinesterase and carbonic anhydrase enzymes inhibition profiles", Journal of Biochemical and Molecular Toxicology, 33(1). doi: ARTN e2223910.1002/jbt.22239

Tugrak, M., Gul, H. I., Bandow, K., Sakagami, H., Gulcin, I., Ozkay, Y., \& Supuran, C. T. 2019. "Synthesis and biological evaluation of some new mono Mannich bases with piperazines as possible anticancer agents and carbonic anhydrase inhibitors". Bioorganic Chemistry, 90, 103095. doi: 10.1016/j.bioorg.2019.103095

Tugrak, M., Inci Gul, H., Sakagami, H., Gulcin, I., \& Supuran, C. T. 2018. "New azafluorenones with cytotoxic and carbonic anhydrase inhibitory properties: 2-Aryl-4-(4- 
hydroxyphenyl)-5H-indeno[1,2-b]pyridin-5ones". Bioorganic Chemistry, 81, 433-439.

Verpoorte, J. A., Mehta, S., Edsall, J. T. 1967. "Esterase activities of human carbonic anhydrases B and C", Journal of Biological Chemistry, 242(18), 4221-4229.

Yamali, C., Gul, H. I., Ece, A., Taslimi, P., \& Gulcin, I. 2018. "Synthesis, molecular modeling, and biological evaluation of 4-[5aryl-3-(thiophen-2-yl)-4,5-dihydro-1Hpyrazol-1-yl] benzenesulfonamides toward acetylcholinesterase, carbonic anhydrase I and II enzymes", Chemical Biology \& Drug Design, 91(4), 854-866.

Yamali, C., Gul, H. I., Ozgun, D. O., Sakagam, H., Umemura, N., Kazaz, C., \& Gul, M. 2017. "Synthesis and Cytotoxic Activities of Difluoro-Dimethoxy Chalcones". Anticancer Agents and Medicinal Chemistry, 17(10), 1426-1433.

Yamali, C., Gul, H. I., Sakagami, H., \& Supuran, C. T. 2016. "Synthesis and bioactivities of halogen bearing phenolic chalcones and their corresponding bis Mannich bases". Journal of Enzyme Inhibition and Medicinal Chemistry, 31(sup4), 125-131. 